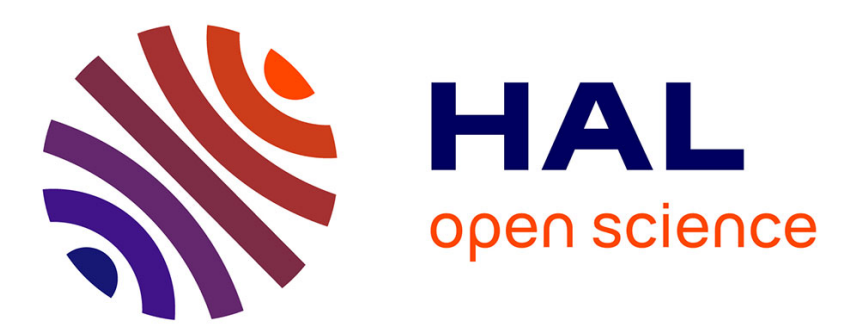

\title{
Range dependent sediment sound speed profile measurements using the image source method
}

\author{
Samuel Pinson, Laurent Guillon, C.W. W Holland
}

\section{To cite this version:}

Samuel Pinson, Laurent Guillon, C.W. W Holland. Range dependent sediment sound speed profile measurements using the image source method. Journal of the Acoustical Society of America, 2013, 134 (1), pp.156-165. 10.1121/1.4809681 . hal-01070963

\section{HAL Id: hal-01070963 \\ https://hal.science/hal-01070963}

Submitted on 4 Dec 2017

HAL is a multi-disciplinary open access archive for the deposit and dissemination of scientific research documents, whether they are published or not. The documents may come from teaching and research institutions in France or abroad, or from public or private research centers.
L'archive ouverte pluridisciplinaire HAL, est destinée au dépôt et à la diffusion de documents scientifiques de niveau recherche, publiés ou non, émanant des établissements d'enseignement et de recherche français ou étrangers, des laboratoires publics ou privés. 


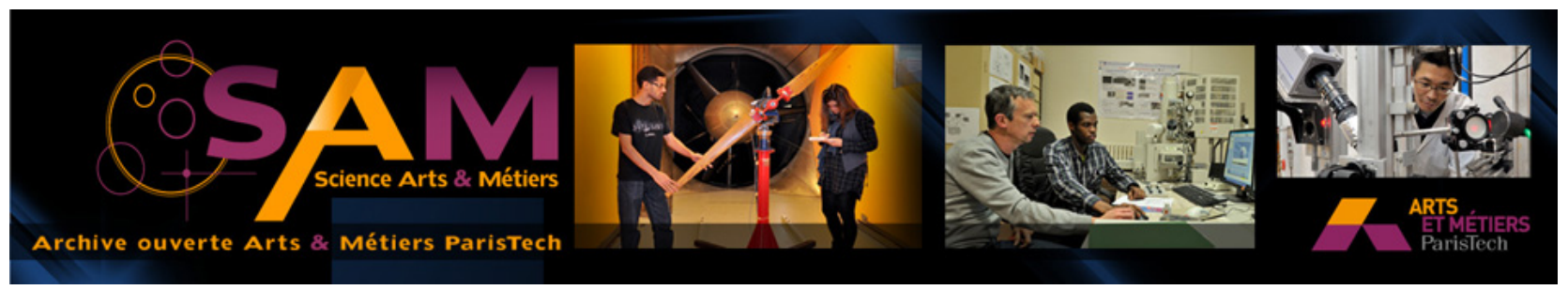

Science Arts \& Métiers (SAM)

is an open access repository that collects the work of Arts et Métiers ParisTech researchers and makes it freely available over the web where possible.

This is an author-deposited version published in: http://sam.ensam.eu

Handle ID: .http://hdl.handle.net/10985/8677

\section{To cite this version :}

Samuel PINSON, Laurent GUILLON, Charles HOLLAND - Range dependent sediment sound speed profile measurements using the image source method - Journal of the Acoustical Society of America - Vol. 134, n¹, p.156-165 - 2013 


\title{
Range dependent sediment sound speed profile measurements using the image source method
}

\author{
S. Pinson ${ }^{\text {a) }}$ and L. Guillon \\ Institut de Recherche de l'Ecole Navale, BCRM Brest, CC 600, F-29240 Brest Cedex 9, France \\ C. W. Holland \\ Applied Research Laboratory, The Pennsylvania State University, State College, Pennsylvania 16804
}

\begin{abstract}
This paper presents a range dependent sediment sound speed profile measurement obtained using the image source method. This technique is based on the analysis of the seafloor reflected acoustic wave as a collection of image sources which positions are linked with the thick-nesses and the sound speed of the sediment stack. The data used were acquired by the NURC in 2009 during the Clutter09 experiment. The equipment used was an autonomous undersea vehicle towing a $1600-3500 \mathrm{~Hz}$ frequency band source and a $32 \mathrm{~m}$ horizontal line array of 32 hydrophones at $12 \mathrm{~m}$ above the seabed. Under the assumption of locally range independent seabed properties, the moving horizontal array provides successive range independent sediment sound speed profiles along a track to obtain the range and depth dependent structure of the seafloor. Two key steps include recovery of the time-varying unknown array shape from the data and spatial filtering of the successive sound speed profiles. A comparison of the image source method result and seismic data along nearly the same $14 \mathrm{~km}$ track indicates that the seabed stratigraphy is correctly mapped by this method.
\end{abstract}

\section{INTRODUCTION}

Most current techniques to characterize the seafloor by acoustics are based on inversion, for example, matched field methods. ${ }^{1}$ In Ref. 2 a new sound speed profile (SSP) measurement method based on the detection of image sources was developed. In that paper the method was validated on simulated data and compared with another method ${ }^{3}$ using real data. The configuration used a vertical array moored on the seafloor and a broadband source towed by a ship. The idea was to consider the geological interfaces as acoustical mirrors on which images of the real source appear. The advantage of imaging the seafloor by searching for point sources is that it becomes possible to determine the seabed structure without an inversion process. The computed SSP is located in the area between the source and the array and is obtained with a low computational cost. In shallow water (water depth under $200 \mathrm{~m}$ ), the seafloor is often highly variable in range. Consequently, using global inversion procedures to obtain a range dependent result may become tricky. A solution of this problem is to consider that the seabed structure is locally range independent and perform successive range independent inversion along a track to obtain a range dependent structure of the seafloor. ${ }^{4,5}$ Based on this idea, the image source method is applied in this paper on data acquired by an horizontal array towed by an autonomous undersea vehicle (AUV) to perform range-dependent seafloor characterization. Moving from a vertical array to a moving horizontal array requires expansion of the method

\footnotetext{
a) Author to whom correspondence should be addressed. Electronic mail: samuelpinson@yahoo.fr
}

presented in Ref. 2. The most challenging issue is that the image technique requires precise knowledge of the array element positions, which are continually changing with time. Another issue is the need for spatial averaging given the small spatial averaging on a single ping $(\approx 20 \mathrm{~m})$. Finally, given the large number of pings (several thousand) a new method for estimating the image source location is required. In this paper numerous SSP measurement have been computed along a $14 \mathrm{~km}$ track to obtain a range dependent result. The paper is organized as follows. First, the data are presented in Sec. II. Then, the image source method and image source detection processing are explained in Secs. III and IV, respectively. The array element localization problem is addressed in Sec. V and the range dependent SSP characterization is finally presented in Sec. VI.

\section{EXPERIMENT DESIGN}

The goal of this experiment was to explore seabed variability along a $14 \mathrm{~km}$ transect on the outer shelf of the Malta Plateau. The experiment was conducted in May 2009 as part of the Clutter09 experiment. A previous survey showed that the bathymetry was smoothly and monotonically varying from about 140 to $130 \mathrm{~m}$ along the track.

The geometry of the experiment is shown in Fig. 1. The receiving array was 32 elements with a spacing of $1.05 \mathrm{~m}$, with the first element $10.38 \mathrm{~m}$ behind the source. More detail on the construction of the array and its tow dynamics are given in Ref. 6. The source was an Ultra Electronics 2-100 MPS cylindrical projector roughly $16 \mathrm{~cm} \times 27 \mathrm{~cm}$, mounted in a prolate spheroidal-shaped tow body. The source transmitted a $1 \mathrm{~s}$ linear frequency modulated pulse $1600-3500 \mathrm{~Hz}$ at a $3 \mathrm{~s}$ pulse repetition rate. The source beampattern is a 


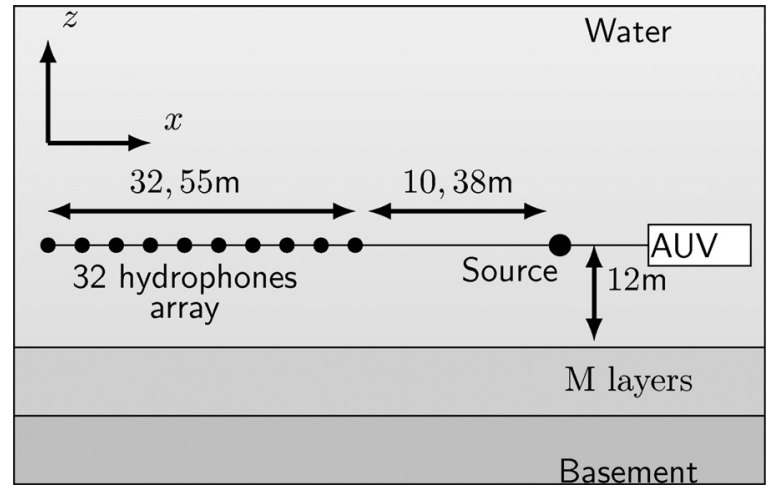

FIG. 1. Sketch of the experiment. The AUV altitude above the seabed is about $12 \mathrm{~m}$ and the receiving array is 32 elements with a spacing of $1.05 \mathrm{~m}$, with the first element $10.38 \mathrm{~m}$ behind the source.

function of angle and frequency, but the processing discussed in the following sections does not require information on absolute or relative levels. The nominal maximum source level at the transducer resonance (about $1800 \mathrm{~Hz}$ ) was $178 \mathrm{~dB} r e 1 \mu \mathrm{Pa} @ 1 \mathrm{~m}$.

The source and receiver were towed by an AUV at a fixed altitude above the seabed of $12 \mathrm{~m}$ at a speed of about $2 \mathrm{kn}$, where the sound source was $2.6 \mathrm{~m}$ behind the AUV propeller. An example of the matched filtered response from the closest hydrophone is shown in Fig. 2. The direct path is clearly visible along with the seabed reflected path with various returns from sub-bottom interfaces. The arrival immediately following the direct path (at $0.011 \mathrm{~s}$ ) is a reflected/ scattered return from the electronics pressure housing inside the AUV. More details about the design of the experiment can be found in Ref. 7 .

\section{IMAGE SOURCE METHOD}

\section{A. Principle}

The image source method is widely used to simulate wave propagation. It models the reflected wave as a wave emitted by the image source located symmetrically behind the reflecting interface. This method is generally used for

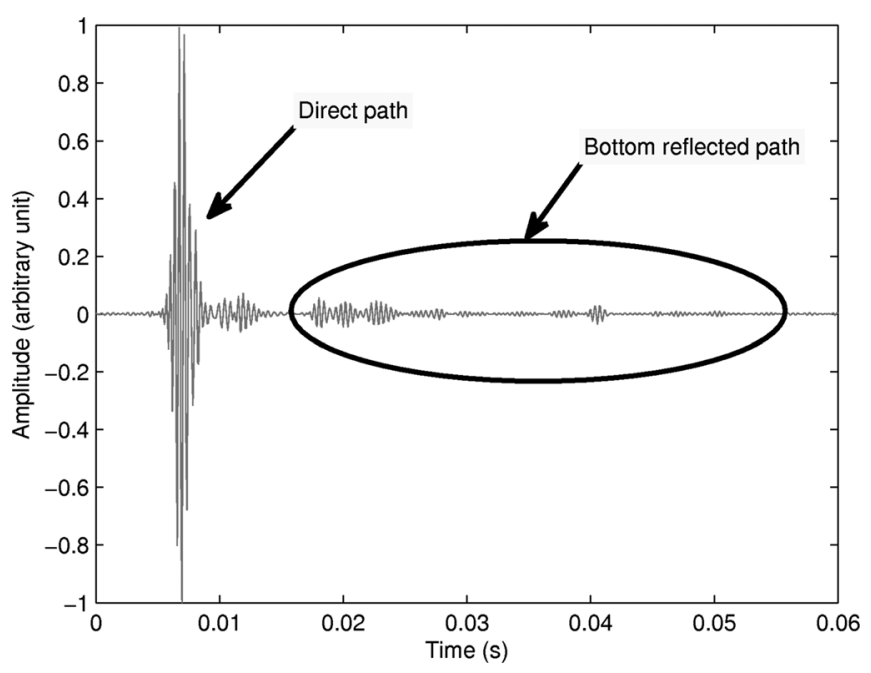

FIG. 2. Example of a recorded signal of the first hydrophone of the array. room acoustics, ${ }^{8}$ propagation in a waveguide, $, 9,10$ or to model the reflection from a half space of an emission from a radar antenna. ${ }^{11}$ In these two last cases, the image source coordinates are complex in order to take into account the angular variations of the reflection coefficient

In Ref. 2, the image source method has been used to invert the SSP of a layered media. This method is based on ray theory and the Born approximation. To model the reflection of an emitted wave as a collection of image sources, the following assumptions are made. First, the water column and the geologic layers are homogeneous, i.e., there is no lateral variability within the measurement window for a single ping. Second, the angle of incidence at an interface is smaller than the critical angle. Third, only the first reflections are taken into account (Born approximation); multiple reflections between interfaces are considered too low in amplitude to interfere with the first ones and be detectable. In this case, each reflection on an interface [Fig. 3(a)] is identified by the receiver as an image source which can be described in an equivalent system: the structure (water + sediment layers) above this interface and its symmetric structure. So, each image is represented in a different equivalent system but, for any given system, the order of the components (water and layer and their symmetric structure) have no consequences on the angle of arrival or on the total travel time. Considering only the travel times and the angles of arrival, it is then possible to merge all the equivalent systems in a single one which contains all the image sources [Fig. 3(b)]. In this system, all the thicknesses are doubled and the images

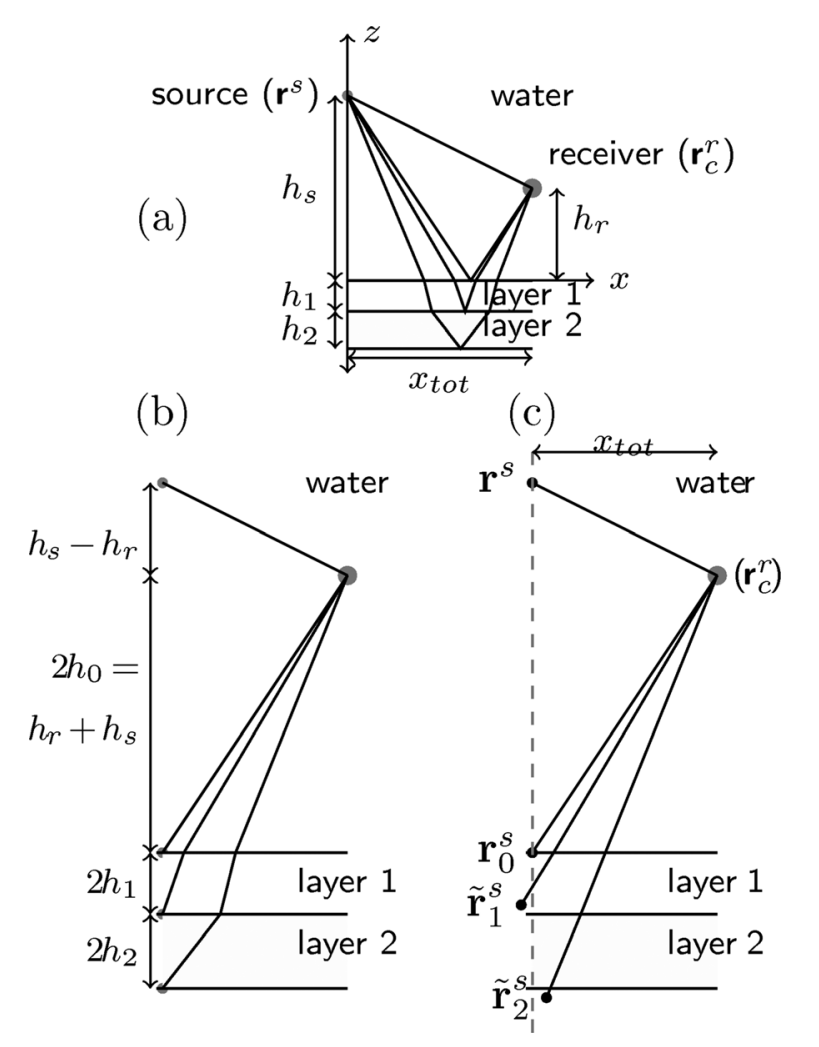

FIG. 3. Modeling of the seafloor with image sources: (a) original configuration, (b) the equivalent system. (c) Image sources not aligned on the vertical source axis due to the water sound-speed everywhere assumption. 
are located on the interfaces and at a zero horizontal offset relative to the source.

\section{B. Inversion algorithm}

The data are used first to produce a map of the image sources. If the layer thicknesses and sound speeds are known, the image sources are located on the vertical source axis because of refraction [Fig. 3(b)]. But since the sound speeds and layer thicknesses are not known, a map can be produced assuming a constant water sound speed everywhere. This assumption leads to an incorrect position of the image sources [Fig. 3(c)]. Even though their locations are wrong, two parameters are correct:

(1) The arrival angles of the waves from the images on the sensor;

(2) The travel times of the waves from the images to the sensor.

The image source method principle then exploits these parameters to obtain the layer thicknesses and sound speeds. In Ref. 2 the SSP is obtained by a recursive alignment of the image sources on the vertical source axis. In this paper, image sources are not aligned on the vertical source axis and the SSP is obtained from the positions of image sources located in a constant sound speed medium. Doing this, the algorithm computation cost is reduced.

The algorithm to estimate the layer thicknesses and sound speeds is recursive. The starting parameters are the heights of source $h_{s}$ and receiver $h_{r}$, the range $x_{t o t}$ and the water sound speed $c_{0}$. Image source coordinates $\mathbf{r}_{i}^{s}$ are numbered from $i=0$, and the real source coordinate $\mathbf{r}^{s}$ has no index. The first image source coordinate $\mathbf{r}_{0}^{s}$ corresponding to the first reflection on the water bottom is correct. Then using the second image coordinate $\mathbf{r}_{1}^{s}$, the first layer thickness and sound-speed can be calculated. This process is repeated for each successive layer.

\section{Sound-speed profile from image source locations}

The coordinate $\mathbf{r}_{l}^{s}$ of the image source $l$ located in a water sound-speed medium yields the parameters $\left[t_{t o t}^{(l)}, \theta_{l}^{(l)}\right]$ characterizing the ray between the image source and the receiver: $t_{\text {tot }}^{(l)}=\left|\mathbf{r}_{c}^{r}-\mathbf{r}_{l}^{s}\right| / c_{0}$ is the travel time, $\mathbf{r}_{c}^{r}$ is the sensor coordinate and $\theta_{0}^{(l)}$ is the angle of arrival of the ray on the sensor (Fig. 4). By convention, the sum of $h_{r}$ (height of the receiver) and $h_{s}$ (height of the source) is called $2 h_{0}$.

The parameters of the ray between the receiver and the image source are

(1) The travel time in the layer $l$,

$$
t_{l}^{(l)}=t_{t o t}^{(l)}-\sum_{p=0}^{l-1} \frac{2 h_{p}}{c_{p} \cos \theta_{p}^{(l)}}
$$

(2) the horizontal distance of the ray in the layer l,

$$
\mathrm{c}_{l} t_{l}^{(l)} \sin \theta_{l}^{(l)}=x_{t o t}-\sum_{p=0}^{l-1} 2 h_{p} \tan \theta_{p}^{(l)}
$$

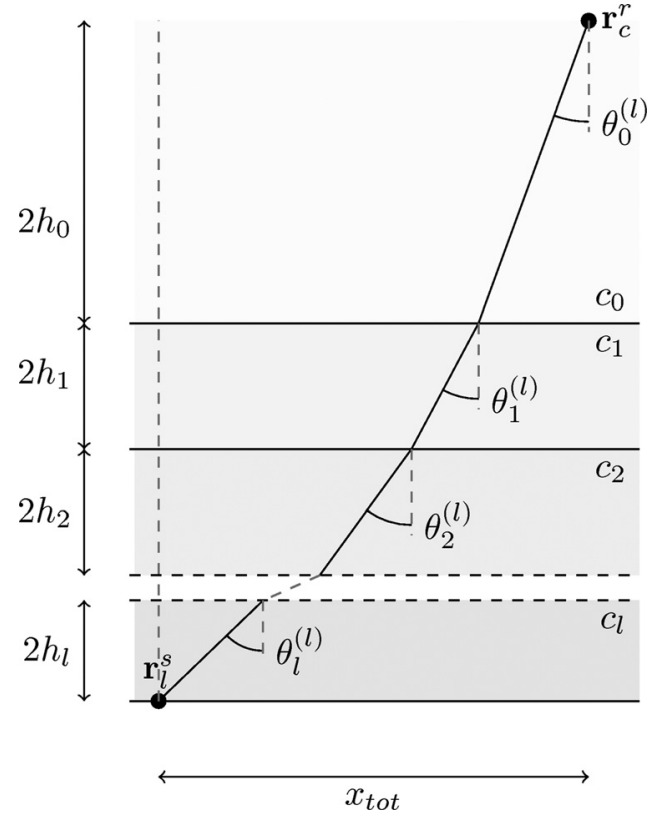

FIG. 4. Refracted ray in a medium with known layer sound speeds and thicknesses.

(3) the Snell-Descartes law,

$$
\sin \theta_{l}^{(l)}=\frac{c_{l}}{c_{0}} \sin \theta_{0}^{(l)},
$$

where $\theta_{p}^{(l)}$ is the incidence angle in the layer $p$ of the ray between $\mathbf{r}_{l}^{s}$ and $\mathbf{r}_{c}^{r}$.

The angle of incidence in each layer is computed:

$$
\theta_{0<i<l-1}^{(l)}=\sin ^{-1}\left[\frac{c_{i}}{c_{0}} \sin \theta_{0}^{(l)}\right]
$$

It is possible to compute the travel time of the ray in the layer $l$ with Eq. (1) and infer the sound speed from Eqs. (2) and (3):

$$
c_{l}^{2}=\frac{c_{0}}{t_{l}^{(l)} \sin \theta_{0}}\left[x_{t o t}-\sum_{p=0}^{l-1} 2 h_{p} \tan \theta_{p}^{(l)}\right] .
$$

Once the sound-speed is computed, the incidence angle in layer $l$ is obtained by

$$
\theta_{l}^{(l)}=\sin ^{-1}\left[\frac{c_{l}}{c_{0}} \sin \theta_{0}^{(l)}\right]
$$

and the thickness of the layer $l$ is obtained by

$$
h_{l}=\frac{1}{2} \mathrm{c}_{l} t_{l}^{(l)} \cos \theta_{l}^{(l)}=\frac{1}{2 \tan \theta_{l}^{(l)}}\left[x_{t o t}-\sum_{p=0}^{l-1} 2 h_{p} \tan \theta_{p}^{(l)}\right] .
$$

The equations are the same as those obtained by Bryan. ${ }^{12}$ However, Bryan's method needs an horizontal array, whereas the method here is more general and can be applied to either horizontal or vertical arrays. ${ }^{2}$ 


\section{ARRAY PROCESSING}

It is possible to deduce the sound-speed profile from the image source locations. These locations can be found by using an array of hydrophones. Then, the ranges between the image sources and the array are given by the wavelet travel times. Because the array is spatially extended, the rays used to compute the sound-speed profile are those coming from the image sources to the center $\mathbf{r}_{n}^{s}$ of the array.

\section{A. Migration function}

The source and its images emit the same pulse $f(t)$ with a different amplitude factor $\beta_{m}$ at the same time. $f(t)$ is a zero phase wavelet for convenience. For $M+1$ point sources located on $\mathbf{r}_{m}^{s}$, the recorded signal on the hydrophone $n$ with coordinate $\mathbf{r}_{n}^{r}$ can be modeled as follow:

$$
\begin{aligned}
s_{n}(t)= & f(t) * g\left(\mathbf{r}^{s}, \mathbf{r}_{n}^{r}, t\right)+\sum_{m=0}^{M-1} \beta_{m} f(t) * g\left(\mathbf{r}_{m}^{s}, \mathbf{r}_{n}^{r}, t\right) \\
& +\eta_{n}(t),
\end{aligned}
$$

where * is the convolution product, $g\left(\mathbf{r}^{s}, \mathbf{r}_{n}^{r}, t\right)$ is the time domain Green function and $\eta_{n}(t)$ is an additive noise spatially white. In this simple model, the Green function is approximated by

$$
g\left(\mathbf{r}_{m}^{s}, \mathbf{r}_{n}^{r}, t\right)=\frac{\delta\left[t-\tau\left(\mathbf{r}_{m}^{s}, \mathbf{r}_{n}^{r}\right)\right]}{\left|\mathbf{r}_{m}^{s}-\mathbf{r}_{n}^{r}\right|},
$$

where $\delta(t)$ is the Dirac delta function and $\tau\left(\mathbf{r}_{m}^{s}, \mathbf{r}_{n}^{r}\right)$ is the travel time between the image source $m$ and the hydrophone $n$.

The analytical signals $s_{n}^{\mathcal{H}}(t)$ are used to get the signal envelopes after focusing:

$$
s_{n}^{\mathcal{H}}(t)=s_{n}(t)+\mathrm{i} \mathcal{H}\left[s_{n}(t)\right],
$$

where $\mathcal{H}\left(s_{n}\right)$ is the Hilbert transform of $s_{n}$.

Image sources are required to be on the vertical source axis. For efficiency, phase-shifts are computed to focus the recorded signals only on a point $\boldsymbol{r}_{\perp}(\theta)$ on the vertical source axis at an incidence $\theta$ from the point of view of the array center (Fig. 5). To focus the recorded signals on the point $\mathbf{r}_{\perp}$ $(\theta)$, each signal is backpropagated by a time $\tau_{n}(\theta)$ corresponding to the travel time between the hydrophone $n$ and the focus point. If an image source is located on $\mathbf{r}_{\perp}(\theta)$, its pulse on the backpropagated signal will be centered around $t=0$. To map the result from the center of the array, a delay $\tau_{c}(\theta)$ is applied which corresponds to the travel time between $\mathbf{r}_{\perp}(\theta)$ and the array center $\mathbf{r}_{c}^{r}$. Calling $d$ the distance between the array center $\mathbf{r}_{c}^{r}$ and a point $\mathbf{r}$ of the image source map (Fig. 5), the process to apply on the signal $s_{n}^{\mathcal{H}}(t)$ of the hydrophone $n$ is:

$$
s_{f n}^{\mathcal{H}}(d, \theta)=D_{n}(\theta) \times s_{n}^{\mathcal{H}}\left[\frac{d}{c}+\tau_{n}(\theta)-\tau_{c}(\theta)\right],
$$

where $\tau_{n}(\theta)=\left|\mathbf{r}_{n}^{r}-\mathbf{r}_{\perp}(\theta)\right| / c_{0}$ and $\tau_{c}(\theta)=\left|\mathbf{r}_{c}^{r}-\mathbf{r}_{\perp}(\theta)\right| / c_{0}$. $D_{n}(\theta)=\left|\mathbf{r}_{n}^{r}-\mathbf{r}_{\perp}(\theta)\right|$ is a spherical geometric divergence compensation.
Finally, image sources are mapped with the following function:

$$
\mathcal{I}^{M}(d, \theta)=\sqrt{\frac{1}{N^{2}} \sum_{n=1}^{N} \sum_{q=1}^{N} s_{f n}^{\mathcal{H}}(d, \theta) s_{f q}^{\mathcal{H} *}(d, \theta)},
$$

where $N^{2}$ is the number of elements of the double sum and the square root is used to obtain a result proportional to a pressure.

The image source map [Fig. 6(a)] with corrected hydrophone coordinates (Sec. VB) lets the real source at $(r$ $\approx 40 \mathrm{~m}, z \approx 0 \mathrm{~m})$ and the images from $(r \approx 40 \mathrm{~m}, z \approx$ $-25 \mathrm{~m})$ to $(r \approx 40 \mathrm{~m}, z \approx-100 \mathrm{~m})$ appear. The first image depth is nearly $25 \mathrm{~m}$ below the real source that is two times the AUV height above the seafloor.

\section{B. Semblance function}

On the map $\mathcal{I}^{M}$, the image source amplitude range is wide and make difficult an automatic detection. To solve this problem, a semblance function ${ }^{13}$ is also mapped:

$$
\mathcal{I}^{\text {semb }}(d, \theta)=\frac{\frac{1}{N^{2}} \sum_{n=1}^{N} \sum_{q=1}^{N} s_{f n}^{\mathcal{H}}(d, \theta) s_{f q}^{\mathcal{H} *}(d, \theta)}{\frac{1}{N} \sum_{n=1}^{N} s_{f n}^{\mathcal{H}}(d, \theta) s_{f n}^{\mathcal{H} *}(d, \theta)} .
$$

With this function, the result is 1 when the focused signals are equal in both amplitude and phase and close to 0 when different.

The result $\mathcal{I}^{M}$ and $\mathcal{I}^{\text {semb }}$ are computed as a function of $d$ and $\theta$. So it is mapped along straight lines starting from the center of the array $\mathbf{r}_{c}^{r}$ with an incidence $\theta$. To map the result as a function of $\mathbf{r}=(x, z)$, the following substitution is applied:

$$
\begin{aligned}
& x=x_{c}^{r}+d \sin \theta \\
& z=z_{c}^{r}-d \cos \theta .
\end{aligned}
$$

The semblance function results in small regions where the focused signals are coherent [Fig. 6(b)] which simplifies image

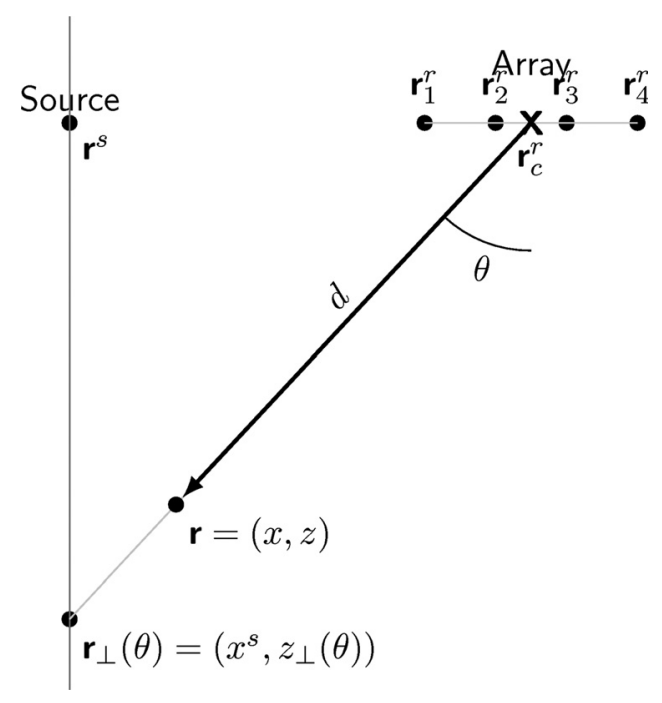

FIG. 5. Sketch of the variables used. 
(a)

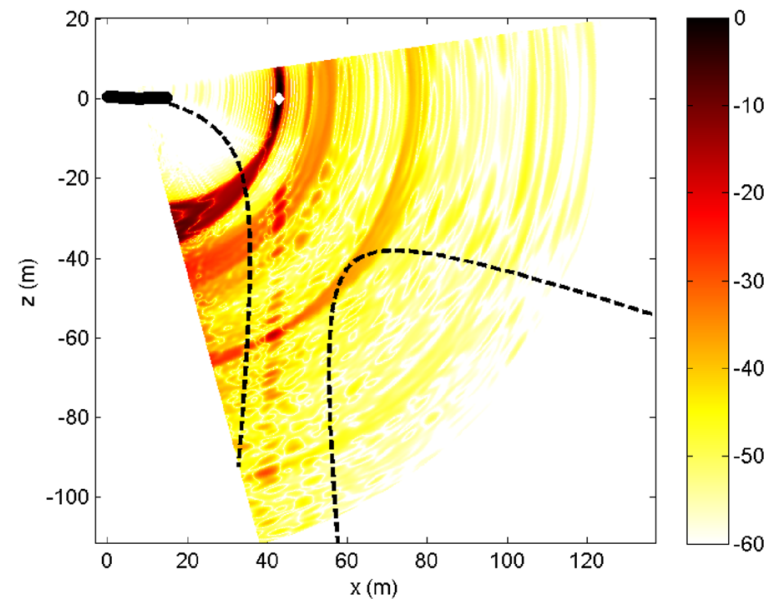

(b)

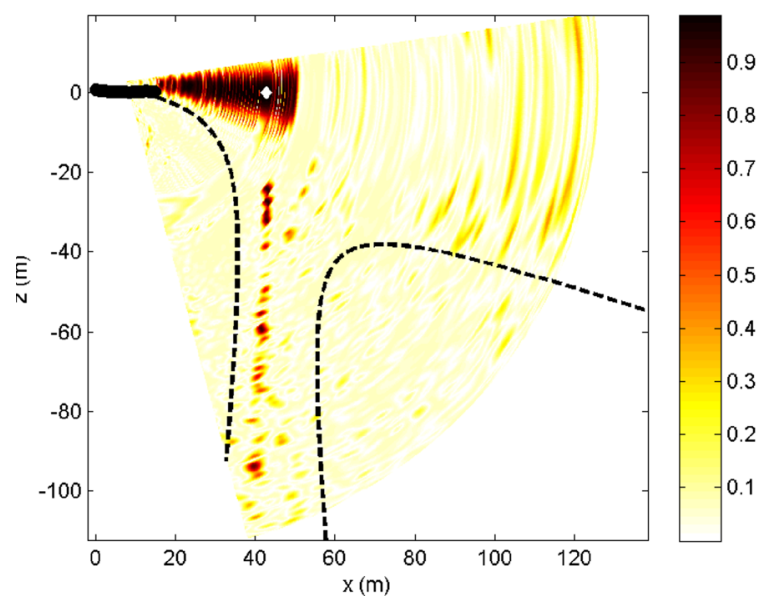

FIG. 6. (Color online) Image sources map computed with hydrophones 18 to 32 for: (a) $\mathcal{I}^{M}$ (in $\mathrm{dB}$ ), (b) $\mathcal{I}^{\text {semb }}$. The white diamond corresponds to the real source location and the black points correspond to the hydrophone locations. The dashed curves correspond to the limits of the depth of field (Sec. IV C).

detection. Applying a threshold on this function, the image source search area is confined to a neighborhood. The image sources locations are found by taking the local maximum of $\mathcal{I}^{M}$.

\section{Depth of field}

Focusing the array on the vertical source axis may lead to missed detections if the image sources are too far from the axis, i.e., the actual sound speed is very far from the "test hypothesis" of the water column sound speed. Image sources are generally close to this axis but it is necessary to check if the depth of field (DOF) is large enough to detect them. The DOF is the area between the near limit $\left(d_{\text {near }}\right)$ and the far $\operatorname{limit}\left(d_{\text {far }}\right):^{14}$

$$
\begin{aligned}
& d_{\text {near }}=d_{\perp}-\frac{\text { res }_{\text {near }} d_{\perp}}{a}, \\
& d_{\text {far }}=d_{\perp}+\frac{\text { res }_{\text {far }} d_{\perp}}{a},
\end{aligned}
$$

where $d_{\perp}$ is the focus distance $\left|\mathbf{r}_{c}^{\mathrm{r}}-\mathbf{r}_{\perp}(\theta)\right|$ and $a=\mid \mathbf{r}_{N}^{\mathrm{r}}$ $-\mathbf{r}_{1}^{r} \mid \sin \theta$ is the apparent array aperture. res $_{\text {near }}$ and res $_{\text {far }}$ are the transverse resolution limits for the near and far limit:

$$
\begin{aligned}
& \operatorname{res}_{\text {near }}=\frac{\lambda d_{\text {near }}}{a}, \\
& \operatorname{res}_{\text {far }}=\frac{\lambda d_{\text {far }}}{a},
\end{aligned}
$$

$\lambda$ is the wavelength in water at the central spectrum frequency.Finally the DOF limits are

$$
\begin{gathered}
d_{\text {near }}=\frac{d_{\perp}}{1+\frac{\lambda d_{\perp}}{a^{2}}}, \\
d_{\text {far }}=\frac{d_{\perp}}{1-\frac{\lambda d_{\perp}}{a^{2}}} .
\end{gathered}
$$

If $\lambda d_{\perp} / a^{2}>1$, the far limit is infinite.

On Fig. 6, one can check that the image sources on the maps $\mathcal{I}^{M}$ and $\mathcal{I}^{\text {semb }}$ are all located between $d_{\text {near }}$ and $d_{\text {far }}$ (yellow curves). For high incidence angles, $d_{\text {far }}$ becomes infinite because the apparent array aperture becomes small.

\section{DIFFICULTIES AND SOLUTIONS}

The configuration used for the experiment leads to two difficulties. First, the emitted signal $f(t)$ is not perfectly known. Second, the array is not perfectly straight because of its flexibility, its buoyancy and/or the AUV motion. To use the image source method, the hydrophone coordinates have to be perfectly known.

\section{A. Emitted signal}

The source emits a $1 \mathrm{~s}$ chirp with a frequency range going from 1.6 to $3.5 \mathrm{kHz}$. The first processing applied on the signals is a pulse compression yielding a resolution of about $75 \mathrm{~cm}$. To estimate the reference source pulse, two hypotheses are made about the array shape. The first is that the difference between the real source-hydrophone distances and the expected distances is weak (lower than a quarter of wavelength at the central frequency of the source spectrum $\approx$ $15 \mathrm{~cm})$. The second is that the distance between the source and the first hydrophone is correct $(10.38 \mathrm{~m})$ because this distance is the least modified by the array deformation. Then, the emitted signal is estimated from the direct path between the source and the hydrophone 1 . To do that, the recorded signal of the hydrophone 1 is backpropagated on the source by a convolution with the inverse green function $g_{0}^{-1}\left(\mathbf{r}^{s}, \mathbf{r}_{1}^{r}, t\right)$ $=\left|\mathbf{r}^{s}-\mathbf{r}_{1}^{r}\right| \times \delta\left(t+\left|\mathbf{r}^{s}-\mathbf{r}_{1}^{r}\right| / c_{0}\right)$ :

$$
s_{b 1}(t)=s_{1}\left(t^{\prime}\right) * g_{0}^{-1}\left(\mathbf{r}^{s}, \mathbf{r}_{1}^{r}, t^{\prime}\right) .
$$

Then, this backpropagated signal is windowed by a semblance function:

$$
\mathcal{I}^{\mathrm{semb}}(t)=\frac{\frac{1}{N^{2}} \sum_{n=1}^{N} \sum_{q=1}^{N} s_{b n}^{\mathcal{H}}(t) s_{b q}^{\mathcal{H} *}(t)}{\frac{1}{N} \sum_{n=1}^{N} s_{b n}^{\mathcal{H}}(t) s_{b q}^{\mathcal{H} *}(t)},
$$

where $s_{b n}$ is the backpropagated signal from the hydrophone $n$. However, this semblance result has rapid fluctuations and 
has to be filtered by convolving with a narrow Gaussian function before applying a threshold. Finally $f(t)$ is given by $s_{b 1}(t)$ by keeping the part of the signal where the filtered semblance value is higher than 0.4 . This 0.4 valued threshold is fixed empirically. Once $f(t)$ is known, a Wiener deconvolution $^{15}$ is done to change the emitted pulse into a zero phase wavelet $f^{\prime}(t)$ :

$$
f^{\prime}(t)=\mathrm{TF}^{-1}\left[\frac{F(\omega) F^{*}(\omega)}{|F(\omega)|^{2}+\varepsilon} W(\omega)\right],
$$

and

$$
s_{n}^{\prime}(t)=\mathrm{TF}^{-1}\left[\frac{S_{n}(\omega) F^{*}(\omega)}{|F(\omega)|^{2}+\varepsilon} W(\omega)\right],
$$

where $\mathrm{TF}^{-1}$ is the inverse Fourier transform, $S_{n}(\omega)$ is the Fourier transform of $s_{n}(t), F(\omega)$ is the Fourier transform of $f(t), W(\omega)$ is a spectrum applied to the emitted pulse and $\varepsilon$ is a small constant fixed empirically. In the following, $f^{\prime}(t)$ and $s_{n}^{\prime}(t)$ are called $f(t)$ and $s_{n}(t)$. After this process, echo maximums correspond to the travel times.

\section{B. Array shape correction}

It is possible to apply a correction on the hydrophone coordinates if the array deformation is weak. This array shape correction is possible from the following data (Fig. 7):

(1) The source coordinates $\mathbf{r}^{s}$ (fixed);

(2) The distances between the hydrophones (1.05 $\mathrm{m})$;

(3) The distances between the source and the hydrophones $d_{1}^{s}$ to $d_{N}^{s}$

(4) The distances between the image 0 and the hydrophones $d_{1}^{i m}$ to $d_{N}^{i m}$.

The distance between the source and the first hydrophone $d_{1}^{s}=10.38 \mathrm{~m}$ and the distance between the hydrophones $(1.05 \mathrm{~m})$ are considered correct. The distances $d_{1}^{s}$ to $d_{N}^{s}$ and $d_{1}^{i m}$ to $d_{N}^{i m}$ are obtained from acoustic measurement. The problem is overdetermined and is solved in fine with a cost function.

\section{Determination of the source/hydrophones and first image/hydrophones distances}

The key step in obtaining the array shape is estimating the source/hydrophone distances $d_{1}^{s}$ to $d_{N}^{s}$ and the first image/ hydrophone distances $d_{1}^{i m}$ to $d_{N}^{i m}$. These distances are first approximated by locating the source and the first image assuming a straight array with the help of the $\mathcal{I}^{M}(\mathbf{r})$ function [Eq. (12)] and the $\mathcal{I}^{\text {semb }}$ (r) function [Eq. (13)]. These distances are obtained with the test coordinates $\tilde{\mathbf{r}}_{1}^{\mathrm{r}}$ to $\tilde{\mathbf{r}}_{N}^{r}$ of a straight horizontal array. A small correction to the distances is obtained by phase analysis of the pulses coming from the source and the first image. To compare the phases of the pulses coming from the source (or the first image), the recorded signals are backpropagated to $\mathbf{r}^{s}$ (or $\mathbf{r}_{0}^{s}$ ) and are windowed with a rectangular function slightly shorter than

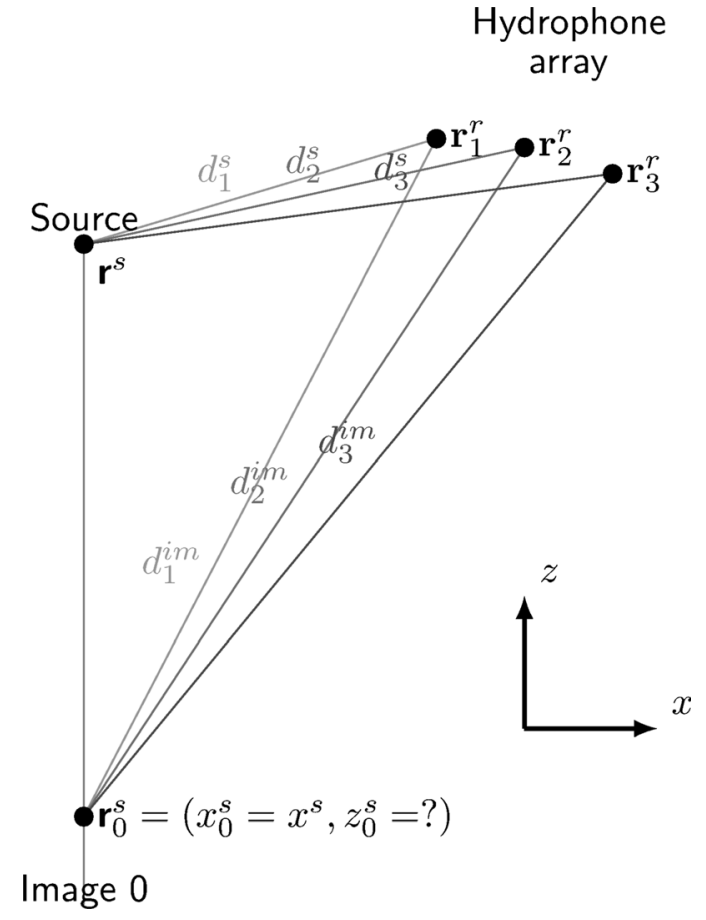

FIG. 7. Distances between the source and the hydrophones and distances between the first image and the hydrophones.

the wavelet $f(t)$ (here $\approx 1 \mathrm{~ms}$ ) and centered around $t=0$. The backpropagated and windowed signal is written:

$$
s_{w n}(t, \mathbf{r})=\left[s_{n}\left(t^{\prime}\right) * g_{0}^{-1}\left(\mathbf{r}, \tilde{\mathbf{r}}_{n}^{\mathrm{r}}, t^{\prime}\right)\right] \Pi_{1 \mathrm{~ms}}(t)
$$

where $\Pi_{1 \mathrm{~ms}}(t)$ is the $1 \mathrm{~ms}$ wide rectangular function and $\mathbf{r}=\mathbf{r}^{s}$ or $\mathbf{r}=\mathbf{r}=\mathbf{r}_{0}^{s}$ if signals are focused on the source or on the image 0 .

The phase shift between each signal $s_{w n}\left(t, \mathbf{r}_{0}^{s}\right)$ and their average provides information to correct the distances between the hydrophones and the image 0 . The phase shift computation is done in the frequency domain at the central frequency of the source spectrum. The corrected distance between the source and the hydrophone $n$ is:

$$
d_{n}^{s}=\left|\tilde{\mathbf{r}}_{n}^{r}-\mathbf{r}^{s}\right|-\frac{c_{0}}{\omega_{c}}\left[\arg S_{w n}\left(\omega_{c}, \mathbf{r}^{s}\right)-\arg F\left(\omega_{c}\right)\right],
$$

and the distance between the first image source and the hydrophone $n$ is:

$$
d_{n}^{i m}=\left|\tilde{\mathbf{r}}_{n}^{r}-\mathbf{r}_{0}^{s}\right|-\frac{c_{0}}{\omega_{c}}\left[\arg S_{w n}\left(\omega_{c}, \mathbf{r}_{0}^{s}\right)-\arg S_{w}\left(\omega_{c}, \tilde{\mathbf{r}}_{0}^{r}\right)\right],
$$

where arg is the argument in radians, $\omega_{c}$ is the pulsation at the central frequency of the source spectrum $F(\omega), S_{w n}(\omega, \mathbf{r})$ and $S_{w}(\omega, \mathbf{r})$ are the Fourier transforms of $s_{w n}(t, \mathbf{r})$ and $s_{w}(t, \mathbf{r})$, respectively.

For the real source, the backpropagated signal phases are compared to $f(t)$ but for the first image, they are compared with the phase of the backpropagated signal average $S_{w}\left(\omega_{c}, \mathbf{r}_{0}^{s}\right)$. This is due to the fact that the reflection on the first interface may change the shape and the phase of $f(t)$ due 
for example to a thin layer, i.e., smaller than the pulse resolution.

\section{Array shape determination}

The receiver $n$ corrected coordinate $\mathbf{r}_{n}^{r}$ is the intersection of the two circles centered on $\mathbf{r}^{s}$ and $\mathbf{r}_{0}^{s}$ with the radius $d_{n}^{s}$ and $d_{n}^{\mathrm{im}}$. At this step, the first image depth $Z_{0}^{s}$ is still unknown. It is determined by minimizing the differences between $\left|\mathrm{r}_{n+1}^{r}\left(z_{0}^{s^{\prime}}\right)-\mathrm{r}_{n}^{r}\left(z_{0}^{s^{\prime}}\right)\right|$ and its theoretical value of $1.05 \mathrm{~m}$ :

$$
z_{0}^{s}=\arg \min _{z_{0}^{s^{\prime}}}\left\{\sum_{n=1}^{N-1}\left[\left|\mathrm{r}_{n+1}^{r}\left(z_{0}^{s^{\prime}}\right)-\mathrm{r}_{n}^{r}\left(z_{0}^{s^{\prime}}\right)\right|-1.05\right]^{2}\right\} .
$$

So, the correction can be done only if $d_{n}^{i m}-\left|\tilde{\mathbf{r}}_{n}^{r}-\mathbf{r}_{0}^{s}\right|$ and $d_{n}^{s}$ $-\left|\tilde{\mathbf{r}}_{n}^{r}-\mathbf{r}_{0}^{s}\right|$ are lower than $\lambda_{c} / 4 \approx 15 \mathrm{~cm}$ where $\lambda_{c}$ is the wavelength at the central frequency of $F(\omega)$. If it is not the case, one can correct the array shape with a low number of receivers and repeat the operation by adding one hydrophone by one hydrophone until the array shape is completely recovered.

An example of a recovered array shape is given on Fig. 8. The real shape deformation is in three dimensions and is recovered considering only two dimensions. However, this two dimensional assumption greatly improves the quality of the image source detection (Fig. 9).

\section{RESULTS}

\section{A. Stability study of the results}

The entire array is not needed to compute the SSP but the number of hydrophones has to be sufficient to locate precisely the image sources. Fifteen hydrophones is a good compromise between resolution and computation cost. ${ }^{16}$ Then it is possible to compute SSP with different subarrays for one transmission of the source. The SSP computed using different subarrays (composed by the hydrophones 1 to 15,2 to $16 \ldots$ and 18 to 32 ) are presented on Fig. 10(a). Depending on the subarray used, the results are very unstable. On Fig. 10 (b) the same instability is found with 20 successive

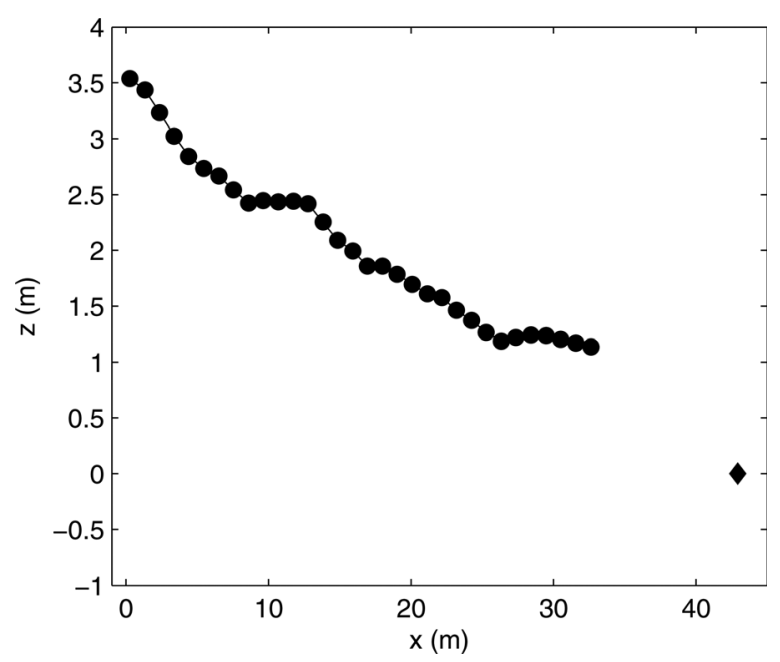

FIG. 8. Example of a recovered array shape (circle). The diamond is the real source coordinate. The vertical exaggeration of the array shape is about 10:1. (a)

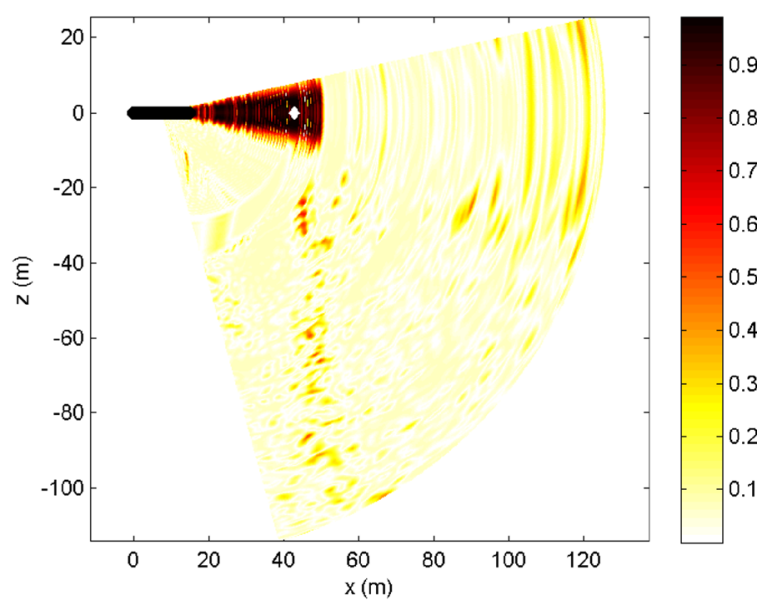

(b)

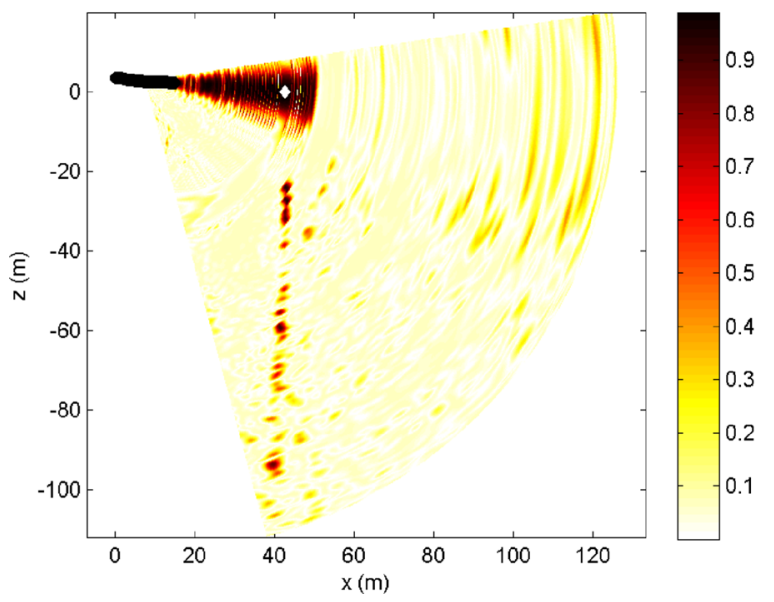

FIG. 9. (Color online) Image sources map computed with hydrophones 18 to 32 for (a) a linear array, (b) the corrected array shape. The white diamond corresponds to the real source location and the black points correspond to the hydrophone locations.

transmissions of the source for a given subarray (composed by the hydrophones 18 to 32). Even though the results are very unstable, stability can be improved through suitable averaging. The averaging can be performed either over the subarrays as in Fig. 10(c) or over multiple source transmissions [Fig. 10(d)]. Averaging over multiple source transmissions appears to give an improved stability.

\section{B. Range dependent characterization}

Using the subarray composed of hydrophones 18 to 32, the sound-speed profiles are computed for the 2693 successive transmissions of the source. In Fig. 11(a) the results are "noisy" but the large-scale sediment structure is clearly appearing. In this figure, $z=0$ corresponds to the sea surface.

As noted in the previous section, averaging over successive transmissions of the source improves the stability of the result. A slightly more sophisticated averaging was applied to the SSP to obtain Fig. 11(a). For the averaging, a two dimensional Gaussian window $w_{2 \mathrm{D}}(\mathbf{x})$ is employed with a $100 \mathrm{~m}$ standard deviation in range and $20 \mathrm{~cm}$ in depth. The 
(a)

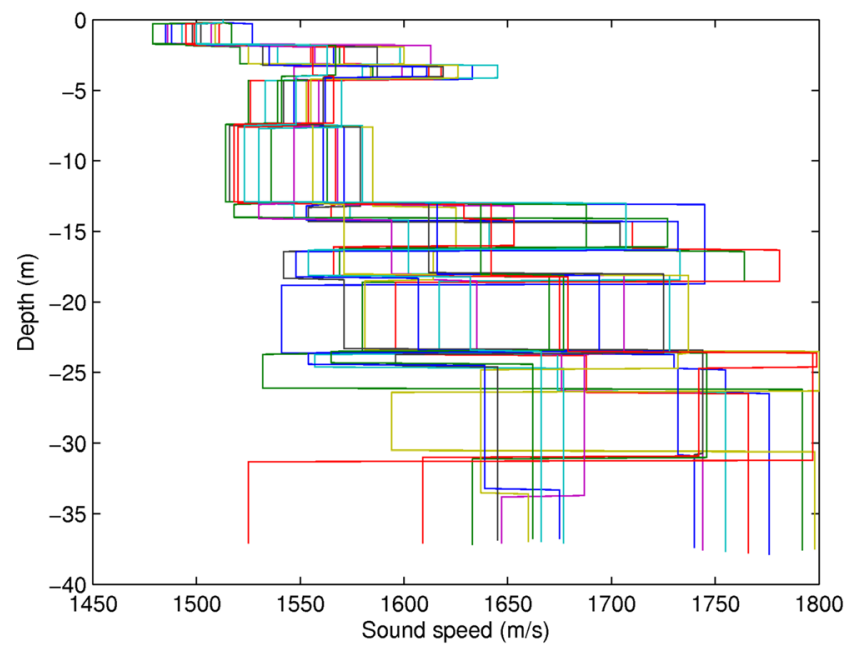

(c)

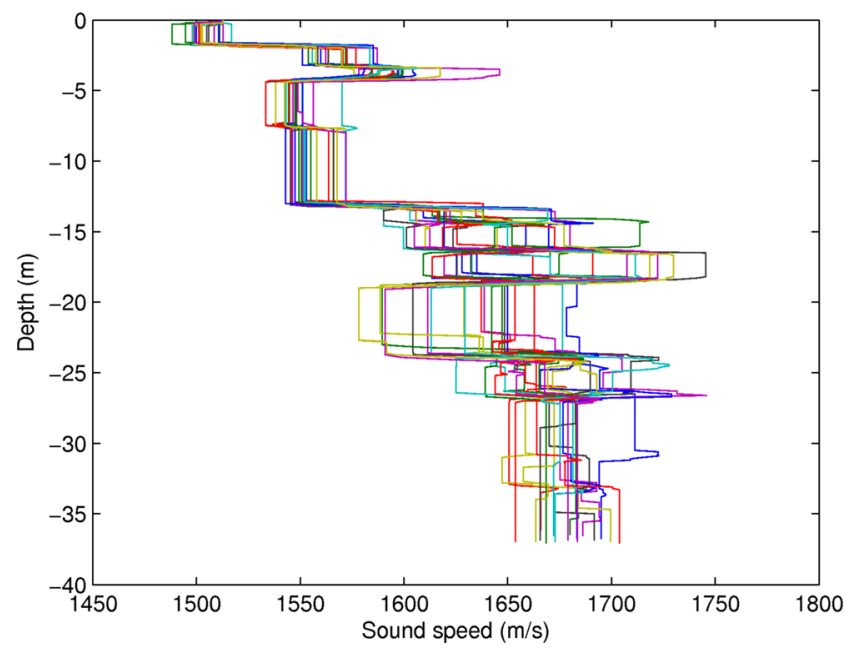

(b)

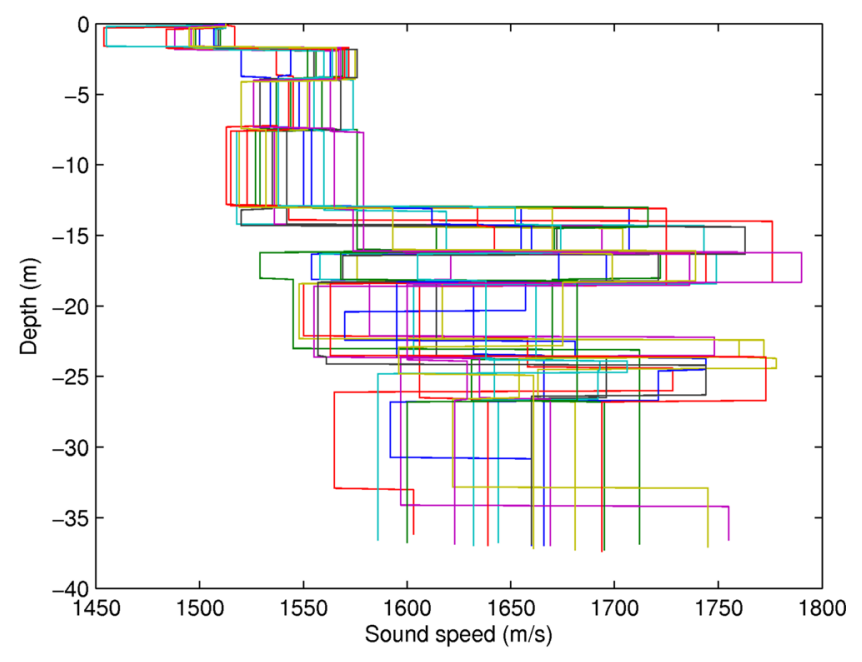

(d)

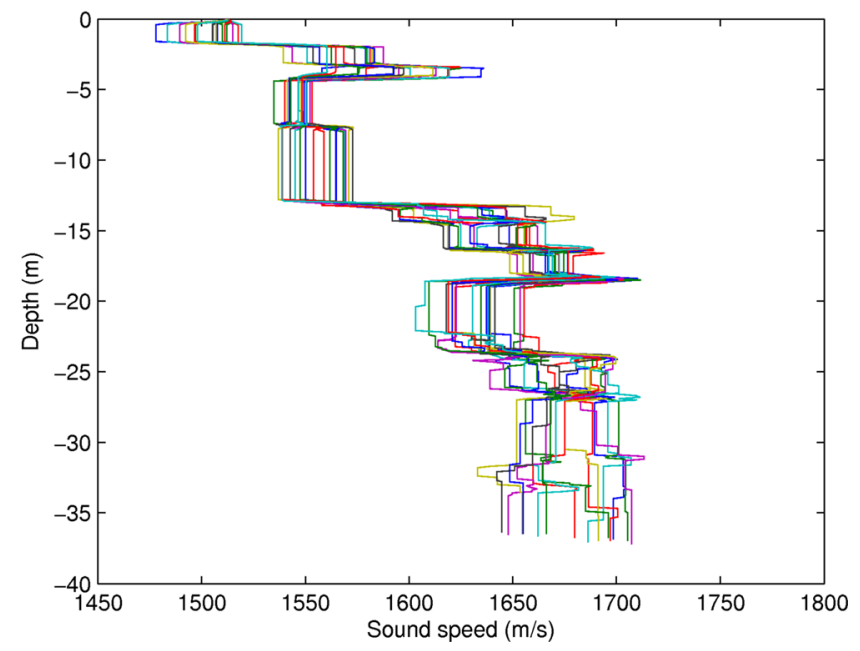

FIG. 10. (Color online) (a) SSP obtained with the subarrays composed of hydrophones 1 to 15,2 to $16, \ldots$ and 18 to 32 for one transmission of the source. (b) SSP obtained for 20 transmissions of the source with the subarray composed of hydrophones 18 to 32 . (c) Averaged SSP over the subarrays for 20 transmissions of the source. (d) Averaged SSP over 20 transmissions for the subarrays composed of hydrophones 1 to 15,2 to $16, \ldots$ and 18 to 32 .

maximum depth of each result can be different from one transmission to another. So, a weight $p(\mathbf{x})=0$ is given where the sound speed are unknown and a weight $p(\mathbf{x})=1$ is given where the sound speed are found. The new notation $\mathbf{x}$ for the coordinates is used instead of $\mathbf{r}$ to avoid confusion with the coordinate system moving with the AUV. Thus, the range dependent filtered SSP is

$$
\bar{c}(\mathbf{x})=\frac{\int w_{2 \mathrm{D}}\left(\mathbf{x}^{\prime}-\mathbf{x}\right) p\left(\mathbf{x}^{\prime}\right) c\left(\mathbf{x}^{\prime}\right) \mathrm{d} \mathbf{x}^{\prime}}{\int w_{2 \mathrm{D}}\left(\mathbf{x}^{\prime}-\mathbf{x}\right) p\left(\mathbf{x}^{\prime}\right) \mathrm{d} \mathbf{x}^{\prime}}
$$

and the standard deviation $\sigma_{c}(\mathbf{x})$ of the filtered SSP is estimated by

$$
\sigma_{c}(\mathbf{x})=\sqrt{\frac{\int w_{2 \mathrm{D}}\left(\mathbf{x}^{\prime}-\mathbf{x}\right) p\left(\mathbf{x}^{\prime}\right)\left[c \mathbf{x}^{\prime}-\bar{c}\left(\mathbf{x}^{\prime}\right)\right]^{2} \mathrm{~d} \mathbf{x}^{\prime}}{\int w_{2 \mathrm{D}}\left(\mathbf{x}^{\prime}-\mathbf{x}\right) p\left(\mathbf{x}^{\prime}\right) \mathrm{d} \mathbf{x}^{\prime}}} .
$$

The integrals of Eqs. (26) and (27) being a convolution product, the Fourier Transform is used for a fast computation

Filtered sound-speed profiles $\bar{c}(\mathbf{x})$ and the standard deviation $\sigma_{c}(\mathbf{x})$ are presented on Figs. 11(b) and 11(c), respectively. Even though the values of $\sigma_{c}(\mathbf{x})$ are relatively large (from 15 to $80 \mathrm{~m} / \mathrm{s}$ ), the sound-speed estimations $\bar{c}(\mathbf{x})$ look realistic. Indeed, for each sediment layer visible on Fig. 11(b), the sound-speed is stable along the $13.5 \mathrm{~km}$ track.

Further averaging can be achieved by averaging over multiple transmissions and multiple subarrays. The soundspeed map presented in Fig. 12(a) is the average of the filtered sound speed map obtained with different subarrays (composed by the hydrophones 6 to 20,7 to $21 \ldots$ and 18 to $32)$. The standard deviation of $\bar{c}(\mathbf{x})$ obtained with the different subarrays is mapped on Fig. 11(d). The reason why the individual SSP errors are so strong is not understood, but if we consider that errors are zeromean, subarray independent, and ping number independent, then the estimate $\bar{c}(\mathbf{x})$ should be reasonably accurate. Indeed The $\bar{c}(\mathbf{x})$ standard deviation values are going from 5 to $25 \mathrm{~m} / \mathrm{s}$. 
(a)

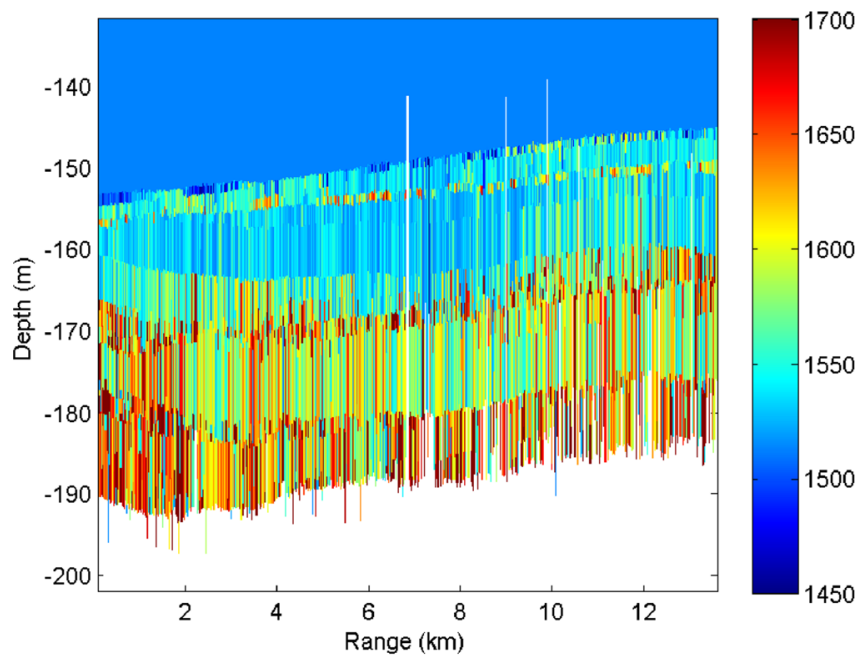

(c)

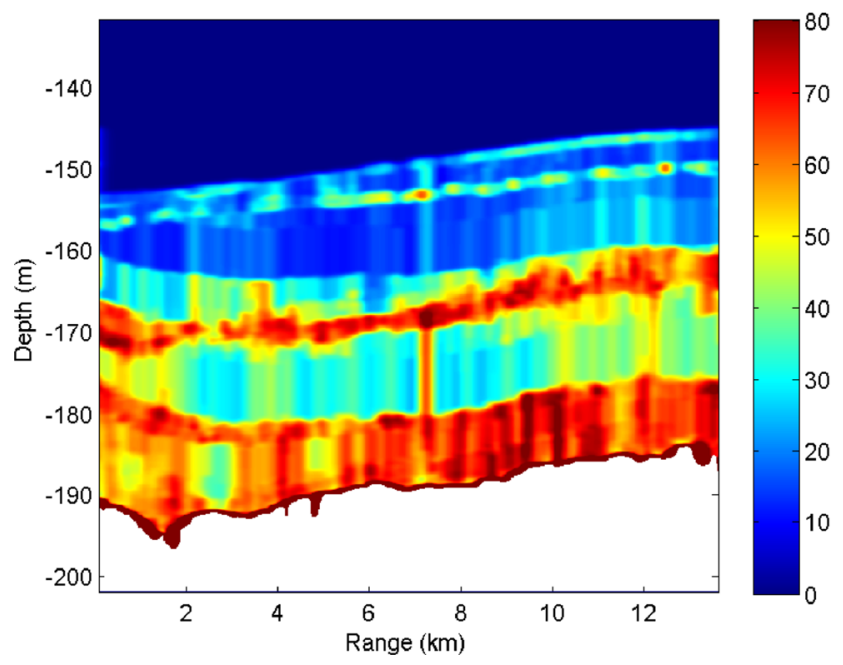

(b)

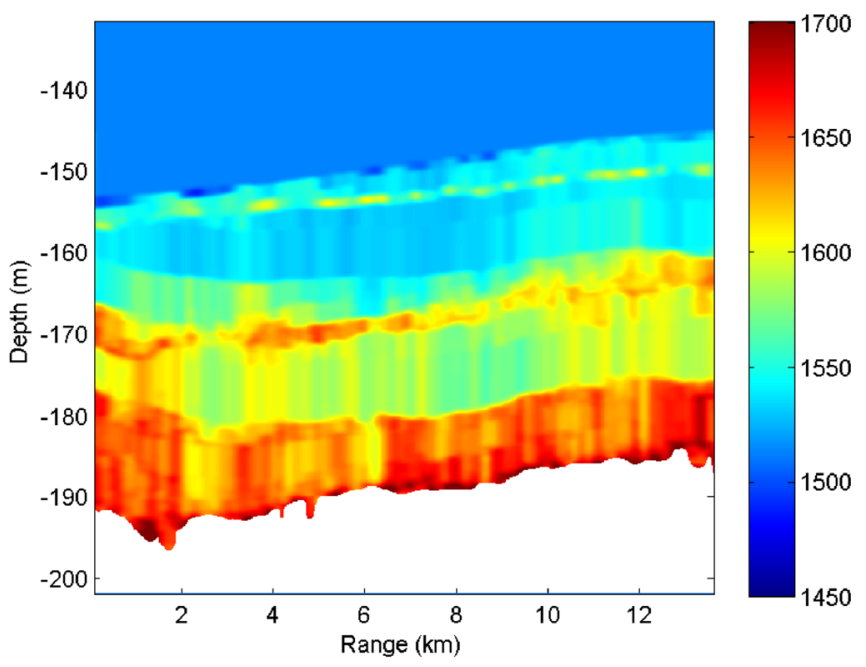

(d)

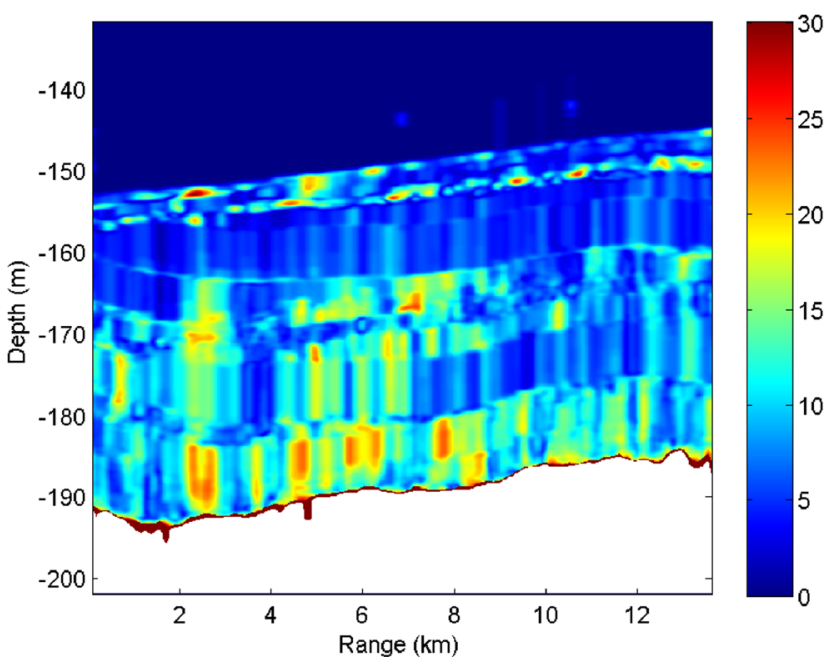

FIG. 11. (Color online) (a) SSP obtained with the subarray composed of hydrophones 18 to 32 for 1 transmission over 4 on the 2693 transmissions of the source. The color scale is fixed from 1450 to $1700 \mathrm{~m} / \mathrm{s}$. (b) Filtered SSP with the subarray composed of hydrophones 18 to 32 for the 2693 transmissions of the source. (c) Standard deviation of the filtered SSP from the subarrays composed of hydrophones 6 to 20,7 to $21 \ldots$ and 18 to 32 . (d) Standard deviation of the filtered SSP from the subarrays composed of hydrophones 6 to 20,7 to $21 \ldots$ and 18 to 32 .

\section{Comparison with the seismic measurement}

In Fig. 12(a), the result is presented with black markers where the interfaces were found by the subarray composed by the hydrophones 18 to 32 . The marker positions are the position of the interface from the image source solution. They make the low sound speed contrasts and layer thickness variations appear more clearly. It is apparent that the low contrast impedance interfaces are not detected for every ping and that markers occur occasionally at random depths. These problems arise because an automated process is applied here to a large and complex data set; for example, it would be impractical to change the image detection threshold of the semblance for each ping. Seismic data were collected by a surface towed boomer along nearly the same track. Some expected differences between the seismic data and the SSP map should be noted. First, the seismic data depth axis is not true depth, but has the usual assumption of a fixed, here $1500 \mathrm{~m} / \mathrm{s}$, sound speed in the sediment whereas the SSP map is plotted with "true" depth, i.e., accounting for the sound speed in each layer. The seismic data have a much larger bandwidth, $10 \mathrm{kHz}$, hence an ostensibly better vertical resolution. However, the seismic source has post-pulse ringing, which reduces the effective resolution and also sometimes leads to apparent but "false" precisely parallel layers. The seismic data have a Fresnel zone roughly three times larger than that of the AUV. However, given the averaging performed on the SSP map, the seismic data is expected to have somewhat better lateral resolution. Given the caveats above, the overall similarity between the SSP map and the seismic data is striking. All of the major layer boundaries in the seismic data are represented in the SSP map. The most complex layers are a series of complex erosional surfaces about $170 \mathrm{~m}$ sub-bottom out to about $8 \mathrm{~km}$ range. These horizons are also detected in the image source method but likely have the greatest uncertainty in the sound speed. 
(a)

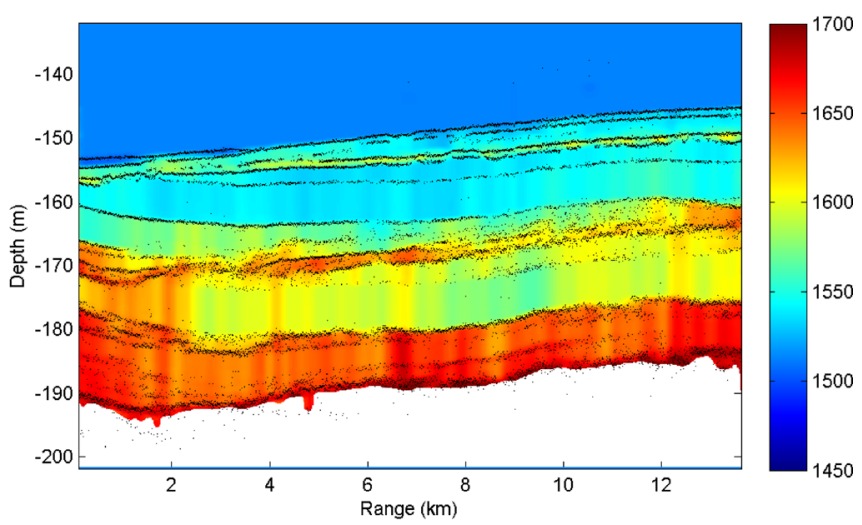

(b)

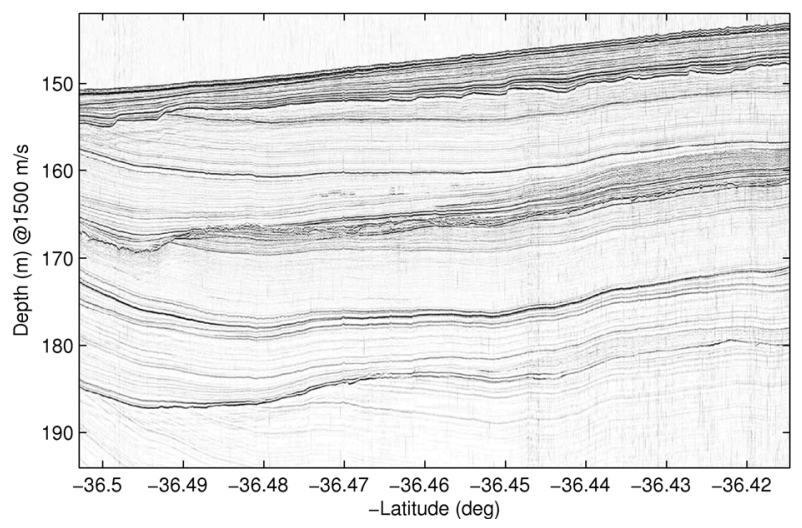

FIG. 12. (Color online) (a) SSP obtained by averaging the filtered SSP from the subarrays composed of hydrophones 6 to 20,7 to $21 \ldots$ and 18 to 32 . The black points correspond to the interface depths found at each transmission of the source. (b) Seismic data along nearly the same track as the AUV. At the track start the two tracks are within a few tens of meters; at the track end the separation is roughly $800 \mathrm{~m}$.

\section{CONCLUSION}

The image source method applied to data acquired by an horizontal array towed by an AUV yields the two dimensional sub-bottom sound speed structure along a $14 \mathrm{~km}$ track. In the data analyzed here, the lateral and vertical resolutions were 200 and $1 \mathrm{~m}$, respectively, with a sub-bottom penetration depth of about $40 \mathrm{~m}$. Inasmuch as the sediment structure is not obtained by doing inversions, the computational requirements are very modest. Comparisons with a seismic profile in the same area indicates that the sediment structure is recovered accurately. There is insufficient data to determine whether or not the sound speeds are correct. The pingto-ping variability is correctly smoothed by filtering, but its origin is not yet understood. A part of this variability is due to the uncertainties of the method itself ${ }^{16}$ but in some areas, the uncertainty appears to be linked to heterogeneities of the seafloor. This could be explored in future work by an analysis of the spatial evolution of the image source semblance values.

\section{ACKNOWLEDGMENTS}

The measurements were made possible by the CLUTTER JRP, a collaboration between ARL-PSU (US), DRDC-A (CA), NATO Undersea Research Center (IT) and NRL (US). The acoustic data were collected during the Clutter09 experiment and the seismic reflection during the Clutter07 experiment. Peter Nielsen was chief scientist on both cruises. The third author gratefully acknowledges the support of the Office of Naval Research, Ocean Acoustics Program.

${ }^{1}$ A. Baggeroer, W. Kuperman, and P. Mikhalevsky, "An overview of matched field methods in ocean acoustics," IEEE J. Oceanic Eng. 18, 401-424 (1993).

${ }^{2}$ S. Pinson and L. Guillon, "Sound speed profile characterization by the image source method," J. Acoust. Soc. Am. 128, 1685-1693 (2010).

${ }^{3} \mathrm{C}$. Holland and J. Osler, "High resolution geoacoustic inversion in shallow water: A joint time and frequency domain technique," J. Acoust. Soc. Am. 107, 1263-1279 (2000).

${ }^{4}$ P. Pignot and N. Chapman, "Tomographic inversion of geoacoustic properties in a range-dependent shallow-water environment," J. Acoust. Soc. Am. 110, 1338-1348 (2001).

${ }^{5}$ M. Siderius, P. Nielsen, and P. Gerstoft, "Range-dependent seabed characterization by inversion of acoustic data from a towed receiver array," J. Acoust. Soc. Am. 112, 1523 (2002).

${ }^{6}$ A. Maguer, R. Dymond, M. Mazzi, S. Biagini, S. Fioravanti, and P. Guerrini, "Slita: A new slim towed array for auv applications," in Acoustics08 (Paris, France, 2008), pp. 141-146.

${ }^{7}$ C. Holland, P. Nielsen, J. Dettmer, and S. Dosso, "Resolving meso-scale seabed variability using reflection measurements from an autonomous underwater vehicle," J. Acoust. Soc. Am. 131, 1066-1078 (2012).

${ }^{8}$ J. Allen and D. Berkley, "Image method for efficiently simulating small room acoustics," J. Acoust Soc. Am 65, 943-950 (1979).

${ }^{9}$ J. Fawcett, "Complex-image approximations to the half-space acoustoelastic Green's function,” J. Acoust. Soc. Am. 108, 2791-2795 (2000).

${ }^{10} \mathrm{~J}$. Fawcett, "A method of images for a penetrable acoustic waveguide," J. Acoust. Soc. Am. 113, 194-204 (2003).

${ }^{11} \mathrm{X}$. Xu and Y. Huang, "An efficient analysis of vertical dipole antennas above a lossy half-space," Prog. Electromagn. Res. 74, 353-377 (2007).

${ }^{12}$ G. Bryan, "The hydrophone-pinger experiment," J. Acous. Soc. Am. 68, 1403-1408 (1980).

${ }^{13} \mathrm{~N}$. Niedell and M. Taner, "Semblance and other coherency measures for multichanel data," Geophysics 36, 482-497 (1971).

${ }^{14}$ J.-P. Pérez, Optique Géométrique et Ondulatoire Avec 200 Exercices et Problèmes Résolus (Geometrical and Physical Optics, 200 Exercises and Problems Solved) (Masson, Paris, France, 1994), Chap. 16.

${ }^{15} \mathrm{M}$. Sacchi, "Statistical and transform methods in geophysical signal processing," available at http://cm-gw.phys.ualberta.ca/ sacchi/saig/book/ (Last viewed April 23, 2012).

${ }^{16}$ S. Pinson, "Caractérisation des fonds marins par la méthode des sources images (seafloor characterization using the image source method)," Ph.D. thesis (Université de Bretagne Occidentale, Brest, France, 2011). 